\title{
Nuevos retos, nuevas estrategias para el futuro: el Campus Virtual de la Universidad Complutense de Madrid
}

\section{New Challenges, new Strategies for the Future: Campus Virtual at Complutense University of Madrid}

\author{
Dra. Amelia Sanz, \\ $\mathrm{U} . \mathrm{C} . \mathrm{M}$. \\ amsanz@filol.ucm.es \\ Dr. José Antonio López Orozco, \\ $\mathrm{U} . \mathrm{C} . \mathrm{M}$. \\ jalodfis.ucm.es
}

\section{RESUMEN}

En este artículo presentamos el Campus Virtual de la Universidad Complutense como una puesta estratégica de nuestra universidad. Se explican los tres grandes retos que rigen del Campus Virtual como son la incorporación al Espacio Europeo de Educación Superior, la internacionalización de la enseñanza, la visibilidad del trabajo que realizan profesores y estudiantes. Finalmente se exponen el conjunto de actuaciones concretas que intentan dar repuestas a estos retos: el desarrollo de metodologías activas y participativas, la integración de la oferta multiplataforma y multiherramienta, las iniciativas de asignaturas y programas en abierto.

PALABRAS CLAVE

b-learning, enseñanza superior, EEES, internacionalización, oCW, competencias,

\section{ABSTRACT}

In this paper we are presenting the Campus Virtual of the Complutense University (Madrid) as a strategical guideline of our university. We identify the three main challenges of our Campus Virtual: the European Espace of High Education, the internationalization od education, the visibility of our teachers' and students' work. Finally, we present some concrete actions we are developing as a response to the challenges: active and collaborative methodologies, integration of platforms and tools in our Campus, open courses and open initiatives.

KEY WORDS

b-learning, high education, EEES, internationalization, oCW, competences,

Decimos que las sociedades occidentales son digitales. Y es que las tecnologías electrónicas han producido un cambio social tanto en los espacios laborales como en los culturales. Sin embargo, la educación ha sido el sector más resistente a la digitalización. Al socaire de la 
transformación científica y tecnológica que vivimos desde los finales del $S$. XX en todas las disciplinas, esperaríamos una renovación didáctica de los modelos de enseñanza y aprendizaje que sin duda está teniendo lugar, pero sin que nos demos bien cuenta de ello. No en vano el libro fue destronado por la pantalla primero como soporte del espectáculo y el arte (con el cine, la televisión y el ordenador), después como almacén del conocimiento y necesariamente ahora como vehículo para la educación. Tenemos que estar preparados ${ }^{1}$.

Las herramientas digitales son hoy una necesidad que ninguna universidad puede ignorar. Y ello a pesar de que la gestión de un conocimiento que quiere ser global, se enfrenta a los obstáculos de la "brecha digital"2, tanto como a los grandes holdings científicos que encierran en sus publicaciones y bases de datos un conocimiento al que sólo se accede con dinero. No nos engañemos: Internet es una red rota en muchas partes del mundo, en algunas disciplinas hasta el 80\% de las bases de datos no son de acceso universal, ningún motor de búsqueda alcanza todos los documentos colgados de la $\mathrm{Red}^{3}$. Pero es deber de las universidades dar respuesta social a estos retos potenciando un acceso al conocimiento que permita la inserción profesional, el aprendizaje adaptado a lo largo de la vida, el progreso para un desarrollo sostenible.

\footnotetext{
1 Amelia Sanz (amsanzafilol.ucm.es) es Profesora Titular del Departamento de Filología Francesa en la Universidad Complutense de Madrid y Directora de la Oficina de Campus Virtual de la misma universidad; José- Antonio López Orozco (jalo@fis.ucm.es) es Profesor Titular del Departamento de Arquitectura de Computadores y Automática de la UCM y Subdirector de la Oficina de Campus Virtual.

2 Recuperemos los datos que nos ofrecen the Internet World Stats: http://www.internetworldstats.com/stats.htm, The Digital Divide.org:http://www.digitaldivide.org/, The Internet Society: http://ww.isoc.org/isoc/ La brecha digital: $\frac{\text { http://www.labrechadigital.org/l }}{3 \text { Es la Deep Web sobre la que conviene insistir con el fin de }}$ alejarnos de la utopía y acercarnos a la denuncia. Basta recordar la entrada que nos facilita Wikipedia en http://en.wikipedia.org/wiki/Deep Web
} 
La Universidad Complutense de Madrid decidió dotarse de un Campus Virtual al servicio de todos sus profesores y estudiantes en 2003 mediante la creación de una Unidad de Apoyo Técnico a la Docencia que ofreciera una herramienta de gestión de asignaturas, cursos y espacios en línea como fue la plataforma WebCT. Desde aquel proyecto piloto con sólo 60 profesores hemos pasado a 4.889 profesores registrados, 89.618 estudiantes, 6.406 espacios creados, que puede alcanzar 70.000 entradas diarias ${ }^{4}$. La UCM es así una universidad presencial que completa sus actividades de enseñanza y de investigación a través del conjunto de herramientas que le ofrece su Campus Virtual.

Esta es la revolución tranquila que está viviendo una universidad con más de 500 años para poner a disposición de todos los miembros de la comunidad universitaria un conjunto de espacios y herramientas en Internet que sirvan de apoyo al aprendizaje, la enseñanza, la investigación y la gestión docente ${ }^{5}$. Así. los profesores-investigadores de la UCM han ido construyendo sus espacios virtuales de forma voluntaria, a su ritmo, a su manera y en su medida ${ }^{6}$.

Conscientes de que el crecimiento exponencial en número de usuarios de estos años ha tocado un techo generacional, ha llegado el momento de realizar una decidida apuesta por la calidad en el proceso de enseñanza-

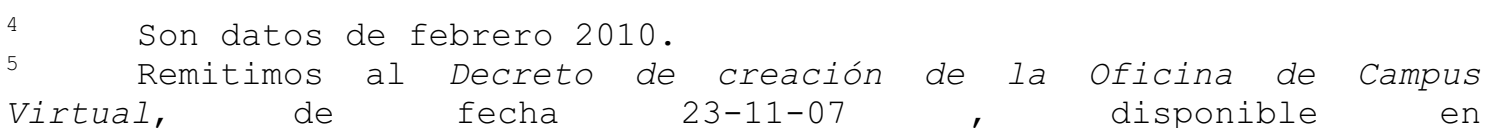

6 Un 30,77\% de los profesores formarían el grupo de aquellos que más utilizan todas las posibilidades de las herramientas, un 49,30\% conforman el grupo de utilización media, mientras que el 19,93\% restante correspondería a los profesores que utilizan tan sólo las funciones básicas de las plataformas (contenidos, correo, gestión de alumnos), según Esther Rodríguez Quintana et al. "Uso y valoración del Campus Virtual de la UCM por parte del profesorado" en Amelia Sanz (ed.), Campus Virtual-UCM 5. Buenas prácticas e indicios de calidad, Madrid, Editorial Complutense, 2009, pp. 316-333. Las experiencias de los profesores de la UCM han sido recogidas a lo largo de las cinco Jornadas que se han celebrado desde 2004 hasta 2009 y que están disponibles https://www.ucm.es/campusvirtual/CVUCM/index.php?ac=jornadas. 
aprendizaje y de trazar con este fin las grandes líneas estratégicas capaces de poner nuestro Campus Virtual al servicio de la Universidad Complutense que queremos para los próximos años como son:

- la incorporación al Espacio Europeo de Educación Superior,

- la internacionalización de la enseñanza,
- la visibilidad del trabajo que realizan profesores y estudiantes.

Para ello, planteamos las siguientes líneas de acción:

- desarrollo de metodologías activas y participativas

- integración de la oferta multiplataforma y multiherramienta

- iniciativas de asignaturas y programas en abierto.

Recogemos aquí así las aportaciones que los profesores de la UCM están haciendo a su Campus Virtual ${ }^{7}$.

\section{Nuestras líneas estratégicas}

La incorporación la Espacio Europeo de Educación Superior $^{8}$ exige la adopción y el desarrollo de sistemas fácilmente comparables de titulaciones que permitan el reconocimiento académico y profesional en toda la UE.

Efectivamente, en Europa, la Educación Superior está recibiendo presiones sin precedentes para adaptarse a las que se consideran necesidades de una sociedad y una economía basadas en el conocimiento. Las cuatro grandes

$7 \quad$ Y que son el objeto de las VI Jornadas de Campus Virtual- UCM, Campus Virtual crece: oportunidades en el EEES y retos para la UCM, 13 de septiembre 2010, UCM (Madrid) (https: / / campusvirtual.ucm.es/SCRIPT/jornadas-581154451 /scripts/serve home)

Para entender qué es el EEES, remitimos a http: //www.ucm.es/pags.php?tp=Políticas\%20específicas\&a=\&d=pags.php?tp =Espacio\%20Europeo\%20de\%20Educación\%20 Superior\&a=directorio\&d=0014785. $\underline{\mathrm{php}}$ 
finalidades que los llamados acuerdos de Bolonia9 plantean son los siguientes:

1. preparación para el mercado laboral

2. preparación para la vida como ciudadanos activos en una sociedad democrática

3. desarrollo personal

4. desarrollo y mantenimiento de una base avanzada de conocimiento.

Ello requiere garantizar la adquisición de las capacidades básicas requeridas al ciudadano ${ }^{10} \mathrm{y}$ trabajar más la adquisición de competencias genéricas y específicas que permitan al estudiante introducirse en el mercado laboral. Por competencia entendemos la combinación dinámica de atributos en torno a conocimientos, habilidades y actitudes ${ }^{11}$, En continuidad con el EEES, la internacionalización de la formación ofrecida por la Universidad Complutense se impone como una necesidad ${ }^{12}$. Efectivamente, dentro de un modelo europeo

9 Bologna Seminar on Qualification Structures in Higher Education in Europe (Copenhagen, Denmark, 27-28 March 2003), organised by Rektorkollegiet, Socrates, and Ministery of Science, Technology and Innovation, $\mathrm{p}$. 1 , in <http://wWw.bolognaberlin2003.de/pdf/Results_copenhagen.pdf>

10 "Les compétences de base comprennent les aptitudes fondamentales que sont la lecture, l'écriture et le calcul, ainsi que la capacité "d'apprendre à apprendre" et les nouvelles compétences définies à Lisbonne, à savoir les compétences en technologies de l'information, les langues étrangères, la culture technologique, l'esprit d'entreprise et les aptitudes sociales." Commission des Communautés Européennes, Réaliser un espace européen de l'éducation et de formation tout au long de la vie, Bruxelles, le 21.11.2001, COM (2001) 678 final, p 27.

11 Bologna Working Group on Qualifications Frameworks (2009): A Framework for Qualifications of the European Higher Education Area, Ministry of Science, Technology and Innovation, Copenhague. Remitimos a Amelia Sanz, "Desarrollo de competencias específicas", in P. García Carcedo, Enseñanza vitual y presencial de las literaturas, Granada, Grupo Editorial Universitario, 2002008 , pp. 15-46.

12 Remito al encuentro The Internationalization of the Higher Education. A foresight exercice for 2020 and beyond que tuvo lugar en la UCM del 19-20 de abril del 2010 (http://www.ucm.es/info/ihe/index.php?option=com content\&view=article\& id=25\&Itemid=15\&lang=en) y, concretamente, las conclusiones en http://www.educacion.es/dctm/eu2010/19y20deabril/madrid-conferencedraftconclusionsihefinal.pdf?document Id=0901e72b800d7f9b 
de educación universitaria, la UCM está en condiciones de proponer modalidades de enseñanza mixta (presencial y virtual) que permitan un aprendizaje de calidad adaptado a las condiciones laborales y de conciliación familiar, con la garantía de equipos docentes capaces de elaborar contenidos en función de las características de los módulos que se van a impartir y de asumir el acompañamiento del estudiante, de forma individual y colectiva, desde que se inicia el proceso formativo hasta que se culmina,

Para ello, el Campus Virtual de la UCM puede ser, en primer lugar, un espacio intermedio entre la enseñanza presencial y la enseñanza a distancia capaz de acercar a los alumnos distantes a través de las herramientas adecuadas y mediante un diseño de contenidos y metodologías adaptados a cada circunstancia. En segundo lugar, nuestro Campus Virtual está llamado a ser el espacio específico y autónomo para nuevas ofertas docentes que permita la extensión de las actividades universitarias de la U.C.M. Porque en tiempo de crisis es tiempo de abrir las puertas.

$Y$ es que una universidad que se quiere excelente ${ }^{13}$ ha de procurar dar visibilidad al trabajo de sus profesoresinvestigadores y de sus estudiantes. El Campus Virtual de la UCM tiene que servir para mostrar la calidad y la especificidad del desarrollo de las clases y los materiales generados por el profesor, de forma que se haga público y reconocido tanto por la sociedad como por la institución el trabajo de los profesores. Efectivamente es mucho el esfuerzo que los profesores dedican a la creación de materiales y al seguimiento de los alumnos mediante herramientas electrónicas, un esfuerzo que no es suficientemente valorado ni reconocido tanto por las instituciones como por la sociedad en general. Con ello será posible presentar modelos de enseñanza mixtos que 
combinen la enseñanza presencial y las herramientas virtuales, tanto como identificar y describir metodologías de calidad que se han demostrado útiles con el fin de compartirlas con toda la comunidad universitaria.

\section{Nuestras líneas de acción}

\section{Desarrollo de metodologías activas y participativas}

El Campus Virtual de la UCM tiene ante sí el reto de implementar y desarrollar de manera efectiva los objetivos y metodologías incluidos en las guías docentes de las asignaturas, para ser más que un lugar donde acumular materiales, lecturas o ejercicios propuestos, incluso más que un instrumento para agilizar la comunicación con los estudiantes, que ya son en sí un acicate, cuando un revulsivo, en la dinámica de enseñanzaaprendizaje.

El reto estriba en explotar al máximo las posibilidades de nuestro Campus Virtual combinando la enseñanza presencial y la no presencial para una docencia basada en la adquisición de competencias: más que un repositorio para poner a disposición del estudiante esquemas, presentaciones de temas, lecturas obligatorias y recomendadas, tienen que contribuir al proceso de preparación, discusión y presentación de trabajos por parte de los alumnos, tienen que apoyarles en sus búsquedas, selecciones y presentaciones de ese conocimiento, tienen que fomentar su capacidad de organización, de planificación y de comunicación en equipo. Ello implica que el profesor ponga al alcance de los estudiantes más materiales y recursos para un trabajo práctico, mientras realiza un seguimiento mayor del estudiante que, por su parte, ha de mantener un esfuerzo continuo y una práctica diaria todavía ajenas a sus hábitos de estudio. Se trata, pues, de vertebrar los conocimientos y la enseñanza práctica, a fin de preparar al alumno para el desempeño de las actividades 
a realizar como profesional y obtener así un aprendizaje significativo, esto es: la capacidad de utilizar el conocimiento en distintos tipos de situaciones que permitan a los profesionales resolver situaciones de la realidad. Así las herramientas de seguimiento y control de alumnos, la posibilidad de creación de grupos de trabajo, generación de informes, etc., permiten al profesor un seguimiento más directo, sin depender de una mediación administrativa. Igualmente, el uso de plataformas constituye una mejora importante de la posibilidad de comunicación mediante correos, foros y blogs, con el fin de abrir espacios de reflexión previa y elaborada mayor que en los debates y discusiones en clase, tanto más que la posibilidad de compartir informaciones con los compañeros de curso puede convertirse en un factor de motivación para indagar en los temas de la asignatura.

Un trabajo sobre competencias requiere un menor peso de las clases magistrales en favor de exposiciones breves y dirigidas, un mayor uso de fuentes por parte del profesor y des estudiante, una participación activa dentro y fuera del tanto para un trabajo individual como colectivo $y$, en consecuencia, una evaluación continua de toda esa actividad.

Para ello, Campus Virtual puede aportar importantes facilidades en la implementación y desarrollo de tareas tales como:

- La resolución de casos prácticos compartidos por varias asignaturas o grupos en Espacios de Trabajo Común, con el fin de potenciar el carácter práctico de una asignatura y subrayar su dimensión profesionalizante, así como la mayor implicación tutorial,

- Los trabajo en grupos ${ }^{14}$ a partir de las herramientas que permiten distribuir y dirigir una tarea a cargo de

14 Para una iniciación a las pautas de un trabajo en grupo, remito a R. Carballo, "Aprender haciendo en grupo. Guía para profesores" en M. C. Chamorro y P. Sánchez Delgado (coor.), Iniciación a la docencia universitaria, Madrid, 2005, pp. 135-160. 
un grupo de estudiantes que pasan a tener su propio espacio virtual de comunicación y de almacenamiento de documentos.

- El uso de tests que promuevan el estudio y el aprendizaje motivando para ello a los estudiantes a través de preguntas que suponen un desafío y un reto intelectual.

- El trabajo colaborativo que persigue la colaboración entre varios individuos a fin de conocer, compartir y ampliar la información que cada integrante de un grupo tiene respecto a un tema determinado, siempre garantizando la intencionalidad y trascendencia de objetivos y finalidades, la reciprocidad y la autovaloración.

- La utilización de blogs ${ }^{15}$ donde el alumno escribe de manera cronológica sus experiencias de aprendizaje y aporta, si lo cree necesario, material relevante, trabajando pues tanto en solitario como con la aportación del grupo.

- La utilización de wikis donde varios integrantes de un grupo realizan una página web cuyo contenido puede ser visto y modificado por cualquier persona con acceso al mismo.

- El foro virtual que se ha convertido en uno de los recursos más utilizados del Campus Virtual, por cuanto que contribuye eficamente al desarrollo del pensamiento crítico, al aprendizaje a través de la interacción social y al desarrollo de capacidades básicas de escritura y de síntesis.

- La vinculación con redes sociales de uso general como Facebook o Twitter de modo que el usuario las encontrara

15 Remitimos al trabajo de Jesús Miguel Flores Vivar y Francisco Esteve Ramírez (ed.), BlogUniversidad. Los blogs en la Universidad como iniciativas prácticas de innovación docente. Análisis, evolución y tendencias, Madrid, Servicio de Publicaciones- Facultad de Ceincias de la Información, 2010. 
integradas dentro de su universo comunicativo, aprovechando además la tendencia del alumnado a crear un Campus Virtual paralelo o al menos un fondo de recursos oficioso.

\section{Integración de la oferta multiplataforma $y$ multiherramienta:}

El Campus Virtual de la UCM no es una plataforma sino un conjunto de plataformas ${ }^{16}$ y herramientas electrónicas puestas al servicio del profesor que han de integrarse a todos los niveles, desde el administrativo al docente pasando por el investigador, en el tejido de nuestra Universidad.

Cuando decimos integrar, queremos designar todos aquellos instrumentos y utilidades que permiten el buen desarrollo de la labor docente e investigadora. Es el Campus Virtual en sí, y no las plataformas disponibles, el que, a través de su portal de servicios (PACV), dispone de todos los datos necesarios para conectar las herramientas de Campus Virtual con el tejido administrativo de la UCM. Dispone, por ejemplo, de datos sobre la planificación docente y sobre la matriculación, a través del Servicio de Gestión Académica-GEA, O de la información del personal docente y de administración y servicios.

De este modo, las plataformas docentes de las que disponemos, como son WebCT, Moodle y Sakai, se encuentran integradas como herramientas dentro del Campus Virtual, que suministra a cada una la información necesaria de usuarios, cursos y otros datos. Además, a través del portal de Campus Virtual se pueden gestionar altas, bajas, pre-inscripciones en asignaturas fusiones de grupos o creación de espacios pra el trabajo en común, en cualquiera de las plataformas utilizadas por el profesor. Estamos en la dirección de una integración mayor con otros servicios de la UCM. Así, se está preparando las utilidades necesarias para poder importar las notas publicadas

16 Para un estado de la cuestión sobre la evolución de las plataformas remito a Covadonga López Alonso, María Matesanz del Barrio (ed), Las plataformas de aprendizaje. Del mito a la realidad, Madrid, Biblioteca Nueva, 2009. Más concretamente, remitimos a los trabajos de Ana Fernández-Pampillón (http://www.ucm.es/info/linguistica/profesores/ana.htm) 
en la plataforma docente con las actas de Gestión Académica, el uso de LDAP y la integración de Campus Virtual en un Portal de Servicios de la UCM en construcción. Por último, en esta vía de la integración, consideramos fundamental hacer posible compartir herramientas de diferentes plataformas entre sí.

El Campus Virtual de la UCM ha adoptado una estructura multiplataforma con el fin de dar respuesta a la variedad de necesidades disciplinares que demandan las veintiséis Facultades y Escuelas que forman la UCM. Esta oferta ha permitido que el docente pueda escoger la plataforma que mejor se adapta a su modo de trabajo y su metodología docente. Por ejemplo el uso de herramientas colaborativas como Blogs o Wikis es mejor en sakai que en Moodle, y ésa es una razón de peso para elegir Sakai por parte de un cierto número de profesores.

Aunque pueda parecer que el uso de dos o tres plataformas incrementa el trabajo en ese mismo orden de magnitud, eso no es cierto si se realiza una cierta planificación. Las herramientas de gestión desarrolladas para altas y bajas de alumnos, creación de asignaturas, etc. se realizan de forma externa y son comunes a todas las plataformas, lo que independiza los servicios ofrecidos de la herramienta final en la que se imparte un curso. Evidentemente, el trabajo es mayor que con una sola herramienta, pues hay que desarrollar modificaciones para cada plataforma, pero no son nuevos desarrollos y los cambios no son grandes, siendo los beneficios aportados son mucho mayores.

Por último, conviene señalar que la administración y gestión de plataformas con los volúmenes de usuarios que se manejan en la UCM provoca una serie de problemas que no son sencillos de tratar; son problemas que, para plataformas con menor número de usuarios y/o cursos, no aparecen, pero a los que nosotros sí tenemos que hacer frente, Es una razón más para distribuir los usuarios en varias plataformas y reducir considerablemente ese problema.

Finalmente, el Campus Virtual de la UCM tiene que integrar herramientas complementarias en su plataformas existentes por 
cuanto que pueden responder a las necesidades de desarrollo del EEES, de internacionalización de los másteres y de mejora de las prácticas de enseñanza mixta (presencial y virtual). Efectivamente, las asignaturas albergadas en nuestro campus Virtual atesoran documentos textuales en cualquier formato, documentos gráficos (desde los mapas geográficos a toda clase de reproducciones artísticas), documentos sonoros con todo tipo de grabaciones y audiovisuales en forma de secuencias de documentales, películas, obras de teatro, filmaciones de clases magistrales o de sesiones de prácticas, juegos y programas informáticos. De hecho ya nuestro gestor multimedia está integrando imágenes y vídeo en las plataformas a través de Complumedia ( http://complumedia.ucm.es/). Pero es necesario integrar en nuestro Campus Virtual un mayor número de utilidades como son:

- herramientas para la organización y diseño de actividades de aprendizaje de forma pautada en el tiempo y adaptada a las necesidades del recorrido del alumno, como es Lams (http://www.lamsinternational.com/)

- herramientas que proponen soluciones de aprendizaje y colaboración en línea y en tiempo real y para la educación síncrona a distancia como Elluminate (http://www.elluminate.com)

- herramientas de autor que permiten generar documentos con una presentación clara y particularmente adaptada a la pantalla, como es Wimba Create (http://www.wimba.com)

- herramientas para la creación de ejercicios interactivos y evaluación en línea como Hot Potatoes (http://hotpot.uvic.ca/)y Respondus (http://www.respondus.com/)

- herramientas de autor gratuitas como XERTE, eXelearning, Courselab, WBTExpress, para la creación de objetos de aprendizaje y su integración en la plataforma;

- repositorios de objetos de aprendizaje, como el creado para el Museo Chasqui (http://macgalatea.sip.ucm.es/web/infoProyecto/present acion.php)

- la inclusión de Mapas conceptuales (http://cmap.ihmc.us/ )

- canales de radio y TV al servicio de la totalidad de la comunidad universitaria en favor de su finalidad 
docente (http://www.tvedu.org/) o canales de youtube dedicados a educación (http: //www.youtube.com/education?b=400)

Son las herramientas que consideramos una universidad presencial y virtual necesita en nuestros días.

\section{Iniciativas de asignaturas y programas en abierto.}

Recordamos que la UCM es una universidad presencial que se ha dotado de un Campus Virtual para prestar apoyo a todas las actividades de enseñanza y aprendizaje. Tras una experiencia de más de seis años, hoy UCM abierta (https://www.ucm.es/campusvirtual/ucmabierta/index.php) quiere mostrar la calidad y el trabajo realizado publicando en abierto un conjunto de asignaturas que los profesores de las distintas áreas disciplinares desarrollan en Campus Virtual.

Con ello nos sumamos a la vía abierta por el Massachussets Institute of Technology con su Open Course Ware-MIT a través del cual se ofrece en abierto el material docente que sus profesores utilizan en las enseñanzas junto con una guía de estudio, una vía que también ha seguido OpenCourseWare-Universia que, a través de medios digitales y en abierto, difunde recursos docentes estructurados en asignaturas de diferentes universidades.

En este contexto, la iniciativa de la UCM tiene personalidad propia: UCM Abierta pretende mostrar en abierto no sólo conjuntos de materiales docentes, sino, particularmente, propuestas y ejemplos de buenas prácticas en una enseñanza universitaria mixta (presencial y virtual) que los profesores han identificado y probado con su trabajo cotidiano y quieren así compartir con la comunidad universitaria. Por ello, en cada una de sus entradas, ofrecemos no sólo el acceso a una asignatura desarrollada en cursos anteriores, sino también una explicitación del 
planteamiento didáctico de la materia junto con una valoración personal por parte del profesor de lo que él considera y prueba como buenas prácticas y, en consecuencia, indicios de calidad.

Efectivamente, con esta iniciativa pretendemos reivindicar el esfuerzo y la calidad de la docencia de los profesores de la UCM, mediante una publicación en abierto que permita una evaluación inter pares, como si de cualquier publicación científica se tratara. UCM Abierta aspira a convertirse así en elemento clave para la acreditación de la calidad como investigación docente que es.

Pero no es la única iniciativa de la U.C.M. - la revista de recursos educativos, REDUCA (http://www.revistareduca.es/), que publica los contenidos docentes de los profesores;

- Las iniciativas de la Facultad de Bellas Artes para exponer los trabajos de sus alumnos y

- Los proyectos de Innovación y Mejora de la Calidad docente financiados por la UCM y en los que muchos grupos de nuestra universidad desarrollan materiales docentes, y herramientas para la docencia en el Campus Virtual, unos materiales que pronto estarán disponibles

En definitiva, el Campus Virtual de la UCM comenzó con unos pocos profesores que vieron la necesidad de utilizar las nuevas tecnologías de la información y comunicación en su docencia y utilizaron para ello un sistema de gestión del aprendizaje como WebCT. Pero en estos momentos, es mucho más. Se ha convertido en un elemento esencial en el desarrollo normal de la docencia, aunque ésta sea presencial, de modo que muchos profesores han expresado su dependencia del Campus Virtual para su labor docente, al 
igual que ocurre con las transparencias, las presentaciones en el PC o la clásica pizarra.

Pero no debemos identificar el Campus Virtual de la UCM como un LMS (Learning Management system) únicamente, puesto que en él se integran un conjunto de servicios que permiten al profesor desarrollar su docencia. Son varias las plataformas que el profesor puede utilizar según sus necesidades y aprovechando los mismos servicios en cada una de ellas.

El Campus Virtual de la UCM es un Campus cada vez es menos 'virtual' y más 'real', un lugar de encuentro de profesores, alumnos y personal de administración y servicios, y lo es gracias a la suma de todos y al esfuerzo de los profesores que, de forma altruista, han creído en las posibilidades que se le ofrecían y han trabajado para ello día a día. 